\title{
The ALADIN interactive sky atlas
}

\section{A reference tool for identification of astronomical sources}

\author{
F. Bonnarel, P. Fernique, O. Bienaymé, D. Egret, F. Genova, M. Louys ${ }^{\star}$, F. Ochsenbein, M. Wenger, and \\ J.G. Bartlett ${ }^{\star \star}$ \\ CDS, Observatoire astronomique de Strasbourg, UMR 7550, 11 rue de l'Université, F-67000 Strasbourg, France
}

Received December 1; accepted December 17, 1999

\begin{abstract}
The ALADIN interactive sky atlas, developed at CDS, is a service providing simultaneous access to digitized images of the sky, astronomical catalogues, and databases. The driving motivation is to facilitate direct, visual comparison of observational data at any wavelength with images of the optical sky, and with reference catalogues.

The set of available sky images consists of the STScI Digitized Sky Surveys, completed with high resolution images of crowded regions scanned at the MAMA facility in Paris.

A Java WWW interface to the system is available at: http://aladin.u-strasbg.fr/.
\end{abstract}

Key words: astronomical data bases: miscellaneous catalogs — atlases — surveys

\section{Introduction}

\subsection{The $C D S$}

The Centre de Données astronomiques de Strasbourg (CDS) defines, develops, and maintains services to help astronomers find the information they need from the very rapidly increasing wealth of astronomical information, particularly on-line information.

Send offprint requests to: D. Egret

e-mail: question@simbad.u-strasbg.fr

* Laboratoire des Sciences de l'Informatique, de l'Image et de la Télédétection, ENSPS, Université Louis Pasteur, F-67000 Strasbourg, France.

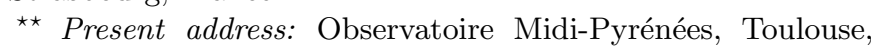
France.
In modern astronomy, cross-matching data acquired at different wavelengths is often the key to the understanding of astronomical phenomena, which means that astronomers have to use data and information produced in fields in which they are not specialists. The development of tools for cross-identification of objects is of particular importance in this context of multi-wavelength astronomy.

A detailed description of the CDS on-line services can be found, e.g., in Egret et al. (1995) and in Genova et al. (1996, 1998, 2000), or at the CDS web site ${ }^{1}$.

\subsection{The ALADIN project}

Several sites currently provide on-line access to digitized sky surveys at different wavelengths: this is, for instance, the case of Digitized Sky Survey (DSS) at STScI (Morrison 1995), and of similar implementations at other sites, providing quick access to cutouts of the compressed DSS images. SKYVIEW at HEASARC (McGlynn et al. 1997) can generate images of any portion of the sky at wavelengths in all regimes from radio to gamma-ray. Some of these services provide simultaneous access to images and to catalogue data. The SKYCAT tool, recently developed at ESO (Albrecht et al. 1997), addressed this concern in the context of the European Southern Observatory scientific environment (in view of supporting future users of the Very Large Telescope); SKyCAT uses a standardized syntax to access heterogeneous astronomical data sources on the network.

AlaDIN has been developed independently by the CDS since 1993 as a dedicated tool for identification of astronomical sources - a tool that can fully benefit from the whole environment of CDS databases and services, and that is designed in view of being released as a multipurpose service to the general astronomical community.

\footnotetext{
${ }^{1}$ Internet address: http://cdsweb.u-strasbg.fr/
} 
AlADIN is an interactive sky atlas, allowing the user to visualize a part of the sky, extracted from a database of images from digitized surveys and observational archives, and to overlay objects from the CDS catalogues and tables, and from reference databases (SIMBAD and NED), upon the digitized image of the sky.

It is intended to become a major cross-identification tool, since it allows recognition of astronomical sources on the images at optical wavelength, and at other wavelengths through the catalogue data. Expected usage scenarios include multi-spectral approaches such as searching for counterparts of sources detected at various wavelengths, and applications related to careful identification of astronomical objects. ALADIN is also heavily used for the CDS needs of catalogue and database quality control.

In the case of extensive undertakings (such as checking the astrometric quality for a whole catalogue), it is expected that ALADIN will be useful for understanding the characteristics of the catalogue or survey, and for setting up the parameters to be adjusted while fine tuning the cross-matching or classification algorithms, by studying a sample section of objects or fields.

A discussion of the usage of such a tool for crossidentification can be found in Bartlett \& Egret (1997), where it is shown how training sets are used to build likelihood ratio tests.

The ALADIN interactive atlas is available in three modes: a simple previewer, a Java interface, and an XWindow interface. We describe here mostly the Java interface which is publicly accessible on the World-Wide Web.

\section{Access modes}

After a long phase of development, (see e.g., Paillou et al. 1994), ALADIN has been first distributed to a limited number of astronomy laboratories in 1997, as an X-Window client program, to be installed on a Unix machine on the user side. The client program interacts with the servers running on Unix workstations at CDS (image server, catalogue server, SIMBAD server) and manages image handling and plane overlays.

The strategy of having a client program on the user side is difficult to maintain on the long run. The WorldWide Web offers, with the development of Java applications (or applets), a way to solve this difficulty. Actually, there is still a client program: this is the Java applet itself, that the user receives from the WWW server. Most current Internet browsers are able to make it run properly, so that the user does not have to install anything special other than an Internet browser.

As a consequence, ALADIN is currently available in the three following modes:

ALADIN previewer: a pre-formatted image server provides a compressed image of fixed size $\left(14.1^{\prime} \times 14.1^{\prime}\right.$ for the
DSS-I) around a given object or position. When an object name is given, its position is resolved through the SImBAD name resolver. Anchors pointing to the previewer are integral part of the World-Wide Web interfaces to the SIMBAD database ${ }^{2}$ and to the CDS bibliographic service ${ }^{3}$. The result page also gives access to the full resolution FITS image for download.

Aladin Java: this is the primary public interface, supporting queries to the image database and overlays from any catalogue or table available at CDS, as well as from SimBAD and NED databases. Access to personal files is not possible (due to security restrictions of the Java language). These restrictions do not apply to the stand-alone version, which can be installed and run on a local Java virtual machine.

Aladin X: The X-Window Aladin client provides most of the functionalities of the ALADIN Java interface, plus more advanced functions, as described below (Sect. 6).

\section{The image database}

\subsection{Database summary}

\section{The ALADIN image dataset consists of:}

- The whole sky image database from the first Digitized Sky Survey (DSS-I) digitized from photographic plates and distributed by the Space Telescope Science Institute (STScI) as a set of slightly compressed FITS images (with a resolution of $1.8^{\prime \prime}$ ); DSS-II is also currently being integrated into the database (see below);

- Images of crowded fields (Galactic Plane, Magellanic Clouds) at the full resolution of $0.67^{\prime \prime}$, scanned at the Centre d'Analyse des Images (MAMA machine) in Paris;

- Global plate views $\left(5^{\circ} \times 5^{\circ}\right.$ or $6^{\circ} \times 6^{\circ}$ according to the survey) are also available for all the plates contributing to the image dataset: these are built at CDS by averaging blocks of pixels from the original scans;

- Other images sets, or user-provided images, in FITS format, having suitable World Coordinate System information in the header (see e.g. Greisen \& Calabretta 1995); this functionality is currently available only for the Java stand-alone version.

\subsection{Building the database contents}

The Aladin project has set up collaborations with the major groups providing digitizations of sky surveys. The original surveys are made of photographic Schmidt plates obtained at Palomar in the North, and ESO/SERC in the

\footnotetext{
2 Internet address: http://simbad.u-strasbg.fr/Simbad

3 Internet address: http://simbad.u-strasbg.fr/biblio.html
} 
South, and covering the whole sky at different epochs and colours (see e.g., MacGillivray 1994).

The database currently includes the first Digitized Sky Survey (DSS-I) produced by the Space Telescope Institute (Lasker 1992), for the needs of the Hubble Space Telescope. To create these images, the STScI team scanned the first epoch (1950/1955) Palomar E Red and United Kingdom Schmidt $J$ Blue plates (including the SERC J Equatorial Extension and some short $V$-band plates at low galactic latitude) with a pixel size of $1.7^{\prime \prime}$ $(25 \mu \mathrm{m})$. The low resolution and a light data compression (factor of 10) permit storage of images covering the full sky on a set of $102 \mathrm{CD}-\mathrm{ROM}$.

DSS-II images in the R-band (from Palomar POSS-II F and UK Schmidt SES, AAO-R, and SERC-ER), scanned with a $1^{\prime \prime}(15 \mu \mathrm{m})$ sampling interval (see Lasker 1994) are gradually being included into the system, and will soon be followed by DSS-II images in the $B$-band (POSS-II J).

In addition, high resolution digitalization of POSS-II, SERC-J, SERC-SR, SERC-I, or ESO-R plates featuring crowded regions of the sky (Galactic Plane and Magellanic Clouds) have been provided by the MAMA facility at the Centre d'Analyse des Images (CAI), Observatoire de Paris (Guibert 1992). Sampling is $0.67^{\prime \prime}$ per pixel $(10 \mu \mathrm{m})$. Currently, these high resolution images cover about $15 \%$ of the sky, and are stored in a juke-box of optical disks, with a capacity of 500 Gigabytes.

\subsection{The image server}

The image server for ALADIN had to be able to deal with various survey data, in heterogeneous formats (uncompressed FITS, compressed JPEG or PMT - see Sect. 5, etc.). For that, an object-oriented design was chosen, allowing an easy manipulation of image calibrations and headers, through the use of object classes. Image compression or decompression, image reconstruction, and in a near future, part of the recalibration, are seen as class methods.

Images are currently divided into subimages of $500 \times$ 500 pixels (DSS-I), $768 \times 768$ pixels $($ DSS-II), or $1024 \times$ 1024 pixels (MAMA).

The 1.5 million subimages are described by records stored in a relational database, encapsulated by several classes of the image management software. When an image of the sky is requested, the original subimages containing the corresponding sky area are retrieved through SQL commands, and the resulting image is built on the fly.

\section{Usage scenarios}

In this section we will focus on describing the usage of the AlaDin Java interface, as it is available now (November 1999).

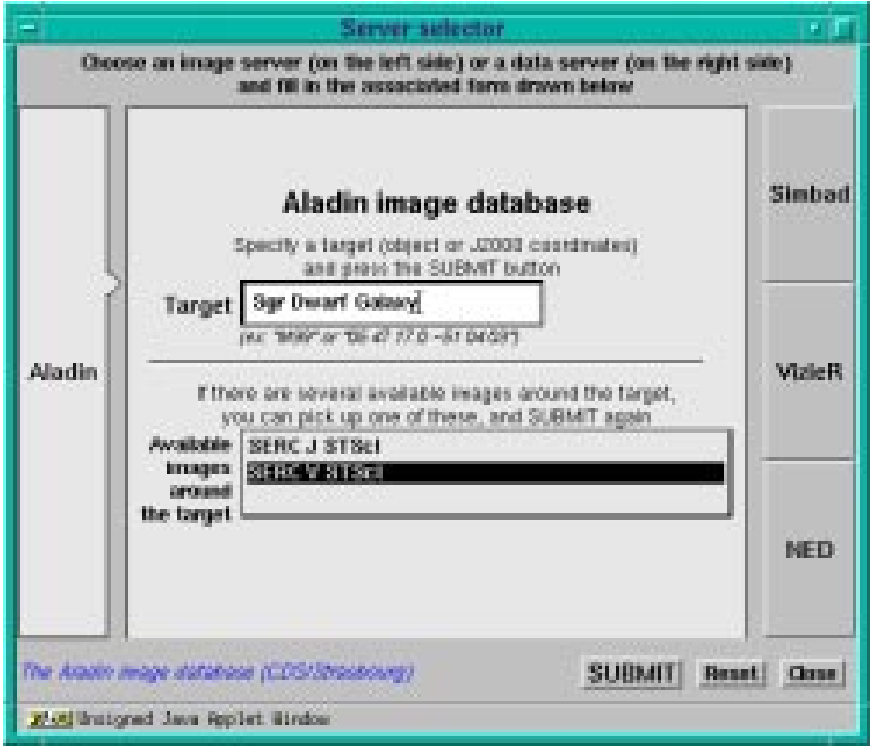

Fig. 1. Example of Images/Data AlAdin query panel

\subsection{Access}

The ALADIN home page is available through the CDS Web server at the following address: http://aladin.u-strasbg.fr/

This site provides access to AlADIN documentation, including scientific reports, recent publications, etc.

\subsection{Query modes}

The typical usage scenario starts with a request of the digitized image for an area of the sky defined by its central position or name of central object (to be resolved by SimBAD). The size of the sky field is determined by the photographic survey used: it is $14.1^{\prime}$ in the case of the DSS-I.

Astrometric information comes from the FITS header of the DSS image, and is generally accurate to the arcsecond (with deviations up to several arcsec. in exceptional cases, on plate edges).

In a subsequent step, the interface, illustrated by Figs. 1 and 2, allows the user to stack several information planes related to the same sky field, to superimpose the corresponding data from catalogues and databases, and to obtain interactive access to the original data.

The possible information planes are the following:

- Image pixels from the ALADIN database of digitized photographic plates (DSS-I, MAMA, DSS-II); functionalities include zooming capabilities, inverse video, modification of the color table;

- Information from the SIMBAD database (Wenger et al. 2000); objects referenced in SIMBAD are visualized by color symbols overlaid on top of the black and white image; the shape and color of the symbols can be modified on request, and written labels can be added for 


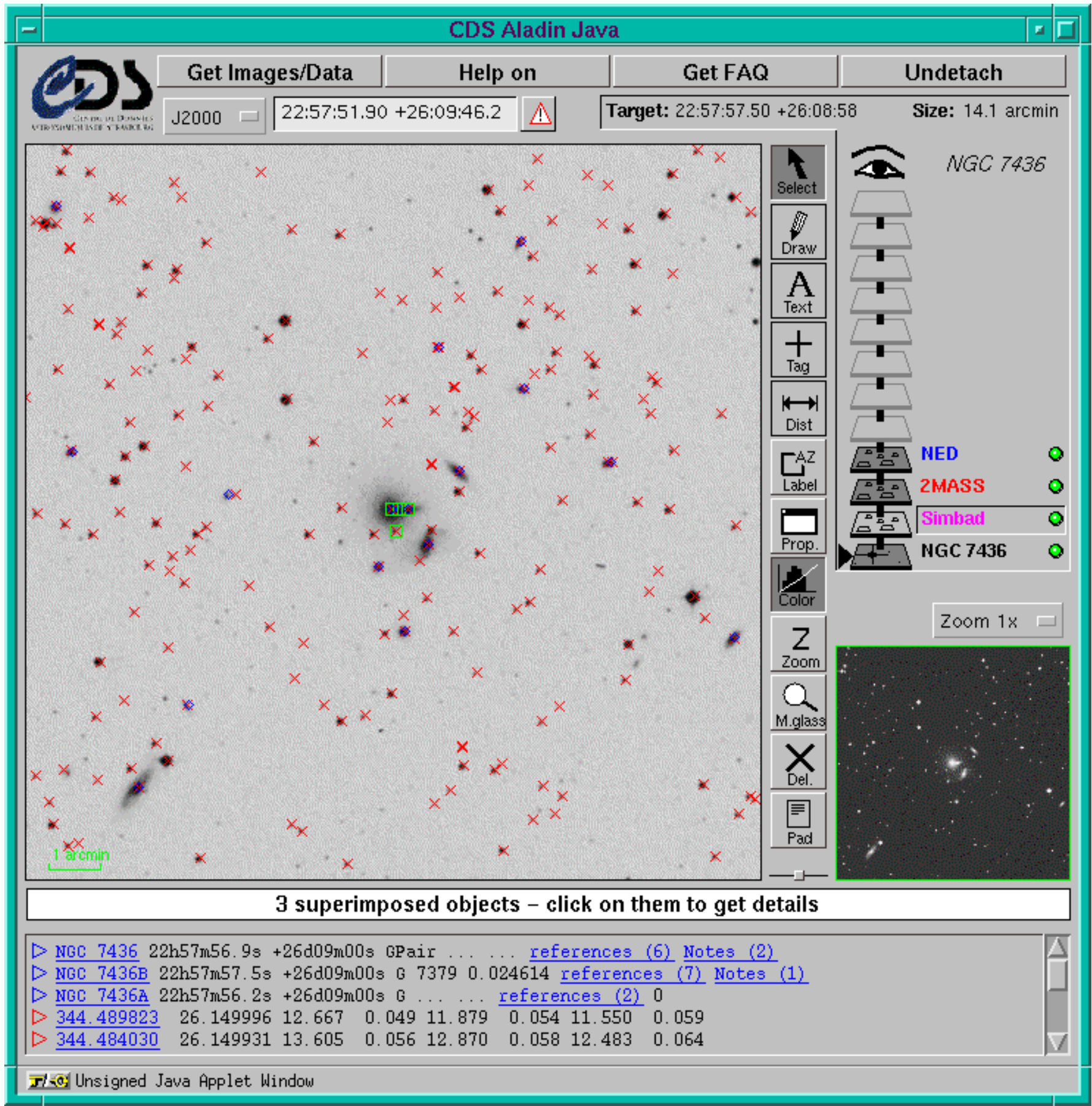

Fig. 2. Example of ALADIN window, with an image centered on NGC 7436, and objects from NED and 2MASS marked by symbols

explicit identification of the objects; these features are also available for all the other information planes;

- Records from the CDS library of catalogues or tables (VIZIER ${ }^{4}$, Ochsenbein et al. 2000); the user can select the desired catalogue from a preselected list including the major reference catalogues such as the Tycho Reference Catalogue (ESA 1997; Høg et al. 1998), GSC
(Lasker et al. 1990), IRAS Point Source Catalog, or USNO A2.0 (Monet 1998); the user can alternatively select the catalogues for which entries may be available in the corresponding sky field, using the VIZIER query mechanism by position (see Sect. 4.3), catalogue name or keyword;

\footnotetext{
4 Internet address: http://vizier.u-strasbg.fr/
} 
- Information from the NED database: objects referenced in the NASA/IPAC Extragalactic Database ${ }^{5}$ (Helou et al. 1991) can also be visualized through queries submitted to the NED server at IPAC;

- Archive images will gradually become available through the corresponding mission logs: Hubble Space Telescope images are currently available (see Fig. 3 for an example), and more archives will follow;

- Local, user data files can also be overlaid, but, because of current limitations of the Java applications, this feature is only available in the stand-alone version, or in Aladin X.

The stack of images and graphics is made visible to the user (under the eye icon, on the right of Fig. 2) so that each plane can be turned on and off. The status of queries is also easily visualized.

For all information planes (SIMBAD, VIZIER, NED) links are provided to the original data. This is done in the following way: when selecting an object on the image, with mouse and cursor, it is possible to call for the corresponding information which will appear in a separate window on the Internet browser. It is also possible to select with the mouse and cursor all objects in a rectangular area: the corresponding objects are listed in a panel on the bottom of the ALADIN window; this list includes basic information (name, position and, when applicable, number of bibliographical references) and anchors pointing to the original catalogue or database.

At any moment the position of the cursor is translated in terms of right ascension and declination on the sky and visualized in the top panel of the ALADIN window. Additional features are available, such as a tool for computing angular distance between marked objects.

The standalone version gives access to additional facilities, not available through the Web, such as printing and saving the images and data.

\subsection{The catalogue server}

The ability to access all VIZIER catalogues and tables directly from ALADIN is a unique feature which makes it an extremely powerful tool for any cross-identification or classification work.

The "Select around target" request relies on a special feature - the genie of the lamp: this is the ability to decide which catalogues, among the database of (currently) over 2600 catalogues or tables, contain data records for astronomical objects lying in the selected sky area. In order to do that, an index map of VIZIER catalogues is produced (and kept up-to-date), on the basis of about ten pixels per square degree: for each such "pixel" the index gives the list of all catalogues and tables which have entries in the field.

\footnotetext{
5 Internet address: http://nedwww.ipac.caltech.edu/
}

When a user hits the button "Select around target", this index is queried and the list of useful catalogues is returned. It is possible, at this stage, either to list all catalogues, or to produce a subset selected on the basis of keywords. Note that, as the index "pixels" generally match an area larger than the current sky field, there is simply a good chance, but not $100 \%$, to actually obtain entries in the field when querying one of the selected tables.

\subsection{Cache}

The images of the 30000 most cited objects in SIMBAD are pre-computed and available on a cache on magnetic disk. For these objects, the image is served much faster than for other objects where the image has to be extracted from the Digitized Sky Survey.

\subsection{Usage statistics}

As the newest service developed by CDS, AlADIN has not yet been widely publicized, and its usage is in a steeply growing phase. Currently about 10000 queries are processed monthly, generating the extraction of more than 5000 images.

\section{Image compression}

Astronomical image compression in the context of ALADIN has been discussed in detail by Louys et al. (1999).

For the AlADiN Java interface and for the AlADIN previewer, the current choice has been to deliver to the user an image in JPEG 8-bit format, constructed from the original FITS images. JPEG is a general purpose standard which is supported by all current Internet browsers. The size of such an image does not exceed $30 \mathrm{kBytes}$, and thus the corresponding network load is very small.

In the near future, the Pyramidal Median Transform (PMT) algorithm, implemented in the MR-1 package (Starck et al. 1996), will be used within AladiN for storing or transferring new image datasets, such as additional high resolution images (see again Louys et al. 1999 for details). The corresponding decompression package is being written in Java code, and could be downloaded on request for use within the Java interface.

\section{Aladin X}

The Aladin X-Window interface is the testbed for further developments. It is currently only distributed for the Unix Solaris operating system. Interested potential users should contact CDS for details. 


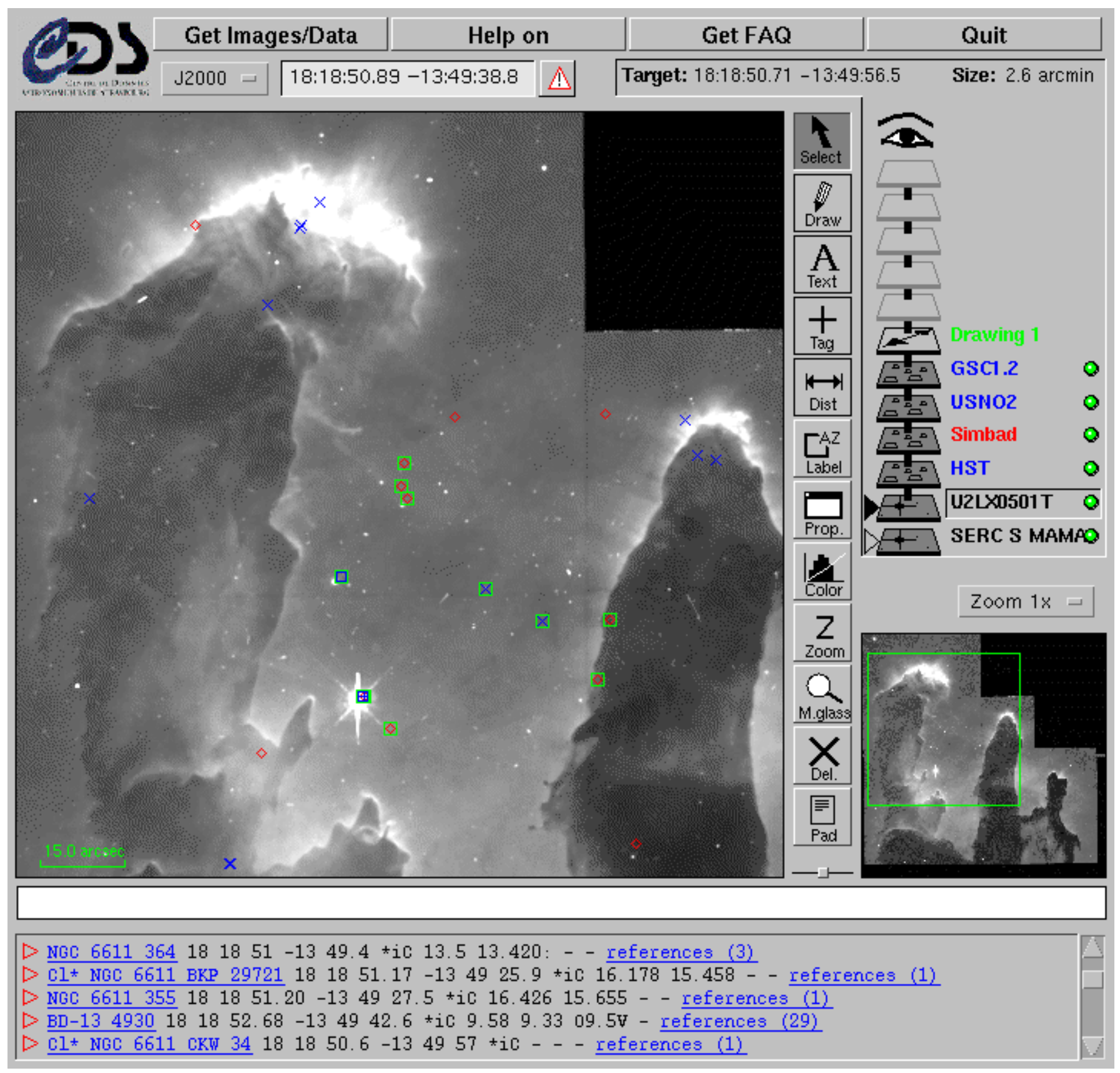

Fig. 3. Example of ALADIN display of a famous image from the HST archive (WFPC2) featuring the Eagle Nebula (M 16). Objects present in Simbad, GSC, and USNO A2.0 are flagged with different symbols. Field size is 2.6 arcmin (full image, right) and 1.6 arcmin (left)

\subsection{Source extraction}

ALADIN X includes a procedure for source extraction. The current mechanism will soon be replaced by SExtractor (Bertin \& Arnouts 1996).

\subsection{Plate calibrations}

While the first level astrometric calibrations are given by the digitizing machines, a second level is being developed that will allow the user to recalibrate the image with a new set of standards taken, for example, from the Tycho Reference Catalogue. The photometric calibrations (surface and stellar) will eventually also be performed within Aladin, by using the Guide Star Photometric Catalogs (GSPC I and II; Ferrari et al. 1994; Lasker et al. 1988).

Users will thus be able to work on the details of local astrometric and photometric plate calibrations in order to extract the full information from the digitized plates. 


\section{Integration of distributed services}

While the CDS databases have followed different development paths, the need to build a transparent access to the whole set of CDS services has become more and more obvious with the easy navigation permitted by hypertext tools. ALADIN has become the prototype of such a development, by giving comprehensive simultaneous access to SIMBAD, the VIzIER Catalogue service, and to external databases such as NED, using a client/server approach and, when possible, standardized query syntax and formats.

In order to be able to go further, the CDS has built a general data exchange model, taking into account all types of information available at the Data Center, known under the acronym of GLU for Générateur de Liens Uniformes - Uniform Link Generator (Fernique et al. 1998).

More generally, with the development of the Internet, and with an increasing number of on-line astronomical services giving access to data or information, it has become critical to develop new tools providing access to distributed services. This is, for instance, the concern expressed by NASA through the AstroBrowse project (Heikkila et al. 1999). A local implementation of this concept is available at CDS (AstroGlu: Egret et al. 1998).

\section{Future developments}

An important direction of development in the near future is the possibility of providing access to images from other sky surveys or deep field observations: obvious candidates are the DENIS (Epchtein 1998) and 2MASS (Skrutskie 1998) near-infrared surveys. The first public point source catalogues resulting from these surveys are already available through ALADIN, since they are included in the VIZIER service. This has already proved useful for validating survey data in preliminary versions of the DENIS catalogue (Epchtein et al. 1999).

The CDS team will also continue to enrich the system functionality. The users play an important role in that respect, by giving feedback on the desired features and user-friendliness of the interfaces.

New developments are currently considered as additional modules which will be incorporated to the general release only when needed, possibly as optional downloads, in order to keep the default version simple and efficient enough for most of the Web applications.

On a longer term, the CDS is studying the possibility of designing data mining tools that will help to make a fruitful use of forthcoming very large surveys, and will be used for cross-matching several surveys obtained, for instance, at different wavelengths. A first prototype, resulting from a collaboration between ESO and CDS, in the framework of the VLT scientific environment is currently being implemented (Ortiz et al. 1999).
Acknowledgements. CDS acknowledges the support of INSUCNRS, the Centre National d'Études Spatiales (CNES), and Université Louis Pasteur.

We are indebted to Michel Crézé who initiated the project while being Director of the CDS, and to all the early contributors to the Aladin project: Philippe Paillou, Joseph Florsch, Houri Ziaeepour, Éric Divetain, Vincent Raclot.

Collaboration with STScI, and especially with the late Barry Lasker, and with Brian McLean, is gratefully acknowledged. We thank Jean Guibert and René Chesnel from CAI/MAMA for their continuous support to the project.

The Digitized Sky Survey was produced at the Space Telescope Science Institute under U.S. Government grant NAG $\mathrm{W}-2166$. The images of these surveys are based on photographic data obtained using the Oschin Schmidt Telescope on Palomar Mountain and the UK Schmidt Telescope.

Java is a registered trademark of Sun Microsystems.

\section{References}

Albrecht M.A., Brighton A., Herlin T., et al., 1997, in Astronomical Data Analysis Software and Systems VI, ASP Conf. Ser. 125, 333

Bartlett J.G., Egret D., 1997, in New Horizons from MultiWavelength Sky Surveys, IAU Symp. 179. Kluwer Academic Publ., p. 437

Bertin E., Arnouts S., 1996, A\&AS 117, 393

Egret D., Crézé M., Bonnarel F., et al., 1995, in Information \& On-line Data in Astronomy, Egret \& Albrecht (eds.). Kluwer Academic Publ., p. 163

Egret D., Fernique P., Genova F., 1998, in Astronomical Data Analysis Software and Systems VII, ASP Conf. Ser. 145, 416

Epchtein N., 1998, in The impact of near-infrared surveys on galactic and extragalactic astronomy, Proc. 3rd. Euroconf. Kluwer Academic Publ., ASSL 230, p. 3

Epchtein N., Deul E., Derriere S., et al., 1999, A\&A 349, 236

ESA, 1997, The Hipparcos and Tycho Catalogues, ESA SP1200

Fernique P., Ochsenbein F., Wenger M., 1998, in Astronomical Data Analysis Software and Systems VII, ASP Conf. Ser. 145,466

Ferrari A., Siciliano E.D., Pizzuti A., et al., 1994, in Astronomy from Wide-Field Imaging, IAU Symp. 161, MacGillivray H.T. \& Thomson E.B. (eds.). Kluwer Academic Publ., p. 301

Genova F., Bartlett J.G., Bienaymé O., et al., 1996, Vistas Astron. 40, 429

Genova F., Bartlett J.G., Bonnarel F., et al., 1998, in Astronomical Data Analysis Software and Systems VII, ASP Conf. Ser. 145, 470

Genova F., Egret D., Bienaymé O., et al., 2000, A\&AS 143, 1 (CDS)

Greisen E.W., Calabretta M., 1995, in Astronomical Data Analysis Software and Systems IV, ASP Conf. Ser. 77, 233

Guibert J., 1992, in Digitized Optical Sky Surveys, MacGillivray H.T. \& Thomson E.B. (eds.). Kluwer Academic Publ., p. 103

Heikkila C.W., McGlynn T.A., White N.E., 1999, in Astronomical Data Analysis Software and Systems VIII, ASP Conf. Ser. 172, 221 
Helou G., Madore B.F., Schmitz M., et al., 1991, in Databases \& On-line Data in Astronomy, Albrecht \& Egret (eds.). Kluwer Acad. Publ., p. 89

Høg E., Kuzmin A., Bastian U., et al., 1998, A\&A 335, 65

Lasker B.M., Sturch C.R., Lopez C., et al., 1988, ApJS 68, 1

Lasker B.M., Sturch C.R., McLean B.J., et al., 1990, AJ 99, 2019

Lasker B.M., 1992, in Digitized Optical Sky Surveys, MacGillivray H.T. \& Thomson E.B. (eds.). Kluwer Academic Publ., p. 87

Lasker B.M., 1994, in Astronomy from Wide-Field Imaging, IAU Symp. 161, MacGillivray H.T. \& Thomson E.B. (eds.). Kluwer Academic Publ., p. 167

Louys M., Starck J.-L., Mei S., Bonnarel F., Murtagh F., 1999, A\&AS 136, 579

MacGillivray H.T., et al., Editors, 1994, Astronomy from Wide-Field Imaging, Postdam, Germany. Kluwer Academic Publ., pp. 1-760

McGlynn T., Scollick K., White N., 1997, in New Horizons from Multi-Wavelength Sky Surveys, IAU Symp. 179. Kluwer Academic Publ., p. 465
Monet D., et al., 1998, The PMM USNO A2.0 Catalogue, US Naval Observatory Flagstaff Station

Morrison J.E., 1995, in Astronomical Data Analysis Software and Systems IV, ASP Conf. Ser. 77, 179

Ochsenbein F., Bauer P., Genova F., 2000, A\&AS 143, 23 (VizieR)

Ortiz P., Ochsenbein F., Wicenec A., Albrecht M., 1999, in Astronomical Data Analysis Software and Systems VIII, ASP Conf. Ser. 172, 379

Paillou Ph., Bonnarel F., Ochsenbein F., Crézé M., 1994, in Astronomy from Wide-Field Imaging, IAU Symp. 161, MacGillivray H.T. and Thomson E.B. (eds.). Kluwer Academic Publ., p. 347

Skrutskie M., 1998, in "The impact of near-infrared surveys on galactic and extragalactic astronomy", Proc. 3rd. Euroconf. Kluwer Academic Publ., ASSL 230, 11

Starck J.-L., Murtagh F., Pirenne B., Albrecht M., 1996, PASP 108, 446

Wenger M., Ochsenbein F., Egret D., et al., 2000, A\&AS 143, 9 (Simbad) 